\title{
La defensa del trabajo de fin de máster por videoconferencia: estrategias y buenas prácticas
}

\section{The master's dissertation viva by video conference: strategies and best practices}

\author{
Cinta Gallent Torres ${ }^{1}$ \\ Fecha de recepción: 05/07/2019; Fecha de revisión: 10/07/2019; Fecha de aceptación: 23/07/2019 \\ Cómo citar este artículo: \\ Gallent Torres, C. (2019). La defensa del trabajo de fin de máster por videoconferencia: estrategias y buenas \\ prácticas. Revista de Innovación y Buenas Prácticas Docentes, 8 (2), 123-135. \\ Autor de correspondencia: cinta.gallent@campusviu.es
}

\section{Resumen:}

Durante la última década, se han publicado numerosos estudios sobre el proceso de elaboración y evaluación de los trabajos de fin de máster (TFM) (Da Cunha, 2016; García \& Martínez-Clares, 2012; González-Calero \& Turégano, 2011), sobre las fases de tutorización y acompañamiento al estudiante (Flores, Martínez, Martínez López, Pascual, \& Sanz, 2013) y sobre las competencias a adquirir tras su finalización (Cabrera, Sangrà \& Rodera, 2012; Serrano \& Pontes, 2015; Viejo \& Ortega Ruiz, 2018). Sin embargo, no resultan tan evidentes las investigaciones que analizan la fase de preparación y defensa pública de estos trabajos, siendo, sin embargo, la que genera más incomodidad entre el estudiantado. El objetivo de este artículo es analizar esta etapa a partir de la experiencia que, como directores de TFM en el Máster Universitario de Formación del Profesorado de la Universidad Internacional de Valencia, hemos tenido durante los últimos años; una experiencia que resultará de interés tanto a directores nóveles que se inician en la tutorización de estos trabajos como a estudiantes que cursan estas asignaturas en modalidad virtual. Se pretende que las estrategias y buenas prácticas aquí compartidas puedan extrapolarse a otras titulaciones e incluso servir de referencia a otras universidades.

Palabras clave: Defensa online, Máster Universitario en Formación del Profesorado, trabajo de máster, tutorización virtual.

\begin{abstract}
:
Over the past decade, many studies have been published on (i) the process of completing and assessing master's dissertations (TFM) (Da Cunha, 2016; García \& Martínez-Clares, 2012; González-Calero, \& Turégano, 2011); (ii) the stages of students' tutoring (Flores, Martínez, Martínez López, Pascual, \& Sanz, 2013); and (iii) the skills students acquire after its completion (Cabrera, Sangrà \& Rodera, 2012; Serrano \& Pontes, 2015; Viejo \& Ortega Ruiz, 2018). Conversely, research analyzing the preparation and public viva phase is quite limited, even though this phase tends to generate the most uncertainty among the students. This article analyzes this phase in the Master's Degree in Teacher Training at the Universidad Internacional de Valencia (VIU) by presenting a series of strategies and best practices implemented over the past years. These initiatives will be of interest to new directors who begin tutoring in this area, as well as to students who study these subjects online. The ideas shared in this article can be applied to other degrees and even be very useful guidelines for other universities.
\end{abstract}

Key Words: Master's degree in teacher training, master thesis, online tutoring, viva video conferencing.

\footnotetext{
${ }^{1}$ Universidad Internacional de Valencia (España), cinta.gallent@campusviu.es; CóDIGO ORCID: 00000002-4260-7594
} 


\section{INTRODUCCIÓN: EL TRABAJO DE FIN DE MÁSTER, REQUISITO OBLIGATORIO DESDE HACE MÁS DE UNA DÉCADA}

Para comprender por qué los estudiantes universitarios deben defender públicamente un trabajo y concluir así sus estudios de postgrado es necesario remitirse al decreto que reguló dicha obligatoriedad.

Se trata, pues, del Real Decreto 1393/2007, de 29 de octubre ${ }^{2}$ que establece las directrices que determinan la puesta en marcha de los estudios de grado, máster y doctorado en las universidades españolas; disposición que prevé modificaciones en los respectivos planes de estudios y requiere que tanto los títulos de grado como los de máster concluyan con la elaboración y defensa pública de un trabajo que permita evaluar las competencias específicas y transversales asociadas con cada titulación. Así pues, los estudiantes de grado que finalicen sus estudios, deberán realizar el denominado trabajo de fin de grado (TFG) y los de máster, el trabajo de fin de máster (TFM), dos materias que, a diferencia del resto de asignaturas de los respectivos ciclos, se desarrollan bajo la supervisión de un tutor que orienta y dirige su elaboración.

Dado que estos trabajos son obligatorios desde hace más de una década, las universidades españolas cuentan con una trayectoria académica que les permite reflexionar sobre su proceso de elaboración, sistema de evaluación, competencias adquiridas y resultados de aprendizaje (Serrano \& Pontes, 2015; Viejo \& Ortega, 2018). Si recordamos, las directrices para la organización, diseño y evaluación de estas materias fueron inicialmente un tanto caóticas, debido a que el ritmo real de implantación de los nuevos planes de estudio fue distinto al establecido por los decretos y las órdenes oficiales (De Pro Bueno, Sánchez \& Valcárcel, 2013; García \& Martínez-Clares, 2012; Sayos, Amador \& Pagés, 2016). Con el tiempo, los centros de educación superior empezaron a regular, a través de normativas internas, los requisitos de elaboración de estos trabajos (extensión, estructura, temática, normas de estilo, etc.), los criterios de evaluación y los protocolos administrativos que permitirían llevar a buen término su implantación (plazos de entrega, asignación de tutores a los estudiantes, gestión de los tribunales de defensa, etc.). Asimismo, el proceso de tutorización y orientación al estudiante fue profesionalizándose y el alumnado fue adaptándose, poco a poco, a las nuevas exigencias académicas en el marco de estas enseñanzas.

Atendiendo al contexto descrito, analizaremos los trabajos de fin de título exigidos por nuestras universidades, concretamente, los trabajos de fin de máster (TFM) que se presentan en uno de los programas de postgrado más solicitados en el ámbito docente: el Máster Universitario de Formación del Profesorado de Educación Secundaria Obligatoria, Bachillerato, Formación Profesional y Enseñanza de Idiomas (en su modalidad virtual). En líneas generales, estos trabajos suelen componerse de dos partes: la elaboración de un proyecto escrito y su defensa pública. Nuestro interés se centra, pues, en la fase final de la asignatura: la presentación y defensa del trabajo realizada a través de videoconferencia; una etapa que genera respeto al estudiantado que se siente evaluado, no sólo por el contenido y la estructura formal de su presentación, la originalidad de las ideas planteadas, la metodología utilizada o la aplicabilidad de su proyecto al ámbito profesional, sino también por la calidad expositiva, capacidad de argumentación y versatilidad ante una situación en la que, de alguna manera, debe hacer valer su proyecto.

\section{EL TRABAJO DE FIN DE MÁSTER EN LA UNIVERSIDAD INTERNACIONAL DE VALENCIA (VIU)}

La Universidad Internacional de Valencia (VIU), centro pionero en España en formación online con clases en directo, ofrece estudios de postgrado desde el año 2010. Desde

\footnotetext{
2 Decreto modificado por el RD 861/2010 de 2 de julio que regula la ordenación de las enseñanzas
} universitarias oficiales. 
este mismo año, los estudiantes pueden cursar el Máster Universitario de Formación del Profesorado tanto en su especialidad de lengua y literatura española como en lengua extranjera, y pueden hacerlo en dos ediciones, abril u octubre.

Mediante esta titulación adquieren la habilitación profesional y la formación necesaria para ejercer la profesión de docentes de Educación Secundaria Obligatoria y Bachillerato, Formación Profesional y Enseñanzas de Idiomas en centros españoles públicos y privados. Para completar estos estudios, deben cursar 60 créditos durante un año académico, lo cual representa una carga de trabajo considerable que debe ser planificada ${ }^{3}$.

Entre las asignaturas obligatorias del máster, el estudiante debe realizar prácticas externas en un centro escolar y presentar una memoria sobre dicha experiencia semiprofesional. En paralelo, debe elaborar y defender su trabajo de fin de máster (TFM), concluyendo así sus estudios de postgrado. Este trabajo final consistirá en la elaboración de una programación docente, la realización de un estudio de caso sobre una problemática identificada en el aula, el diseño de materiales de carácter innovador o de recursos dirigidos a atender a alumnos con necesidades educativas especiales o altas capacidades. Dicho trabajo se elabora durante el segundo semestre y se presenta, en doble convocatoria, al final del máster, una vez superados todos módulos teóricos del programa.

Si bien todas las asignaturas son especialmente interesantes en la formación del futuro docente, el TFM le permite no solo elaborar un proyecto personal, original e inédito sobre un tema relacionado con los contenidos teórico-prácticos del máster, sino también aplicar las competencias específicas y transversales adquiridas a lo largo del título (Cabrera et al., 2012; Hernández-Leo et al., 2013). Posteriormente dicho proyecto será defendido ante un tribunal evaluador compuesto por tres docentes especialistas nombrados por la universidad, prueba que deberá también ser superada para la expedición del título del máster.

Se requiere, pues, al estudiante que realice un trabajo individual, pero dirigido. En este sentido, se le asigna un profesor tutor que supervisa su proyecto, dinamiza el proceso de aprendizaje y vela porque alcance el rigor y la calidad científica propia de estos niveles educativos. Dicho tutor le acompañará a lo largo del proceso de elaboración del trabajo escrito y la defensa del mismo.

Dado que esta asignatura no es específica de este máster, sino que es común a todos los másteres ofrecidos por la universidad, las directrices básicas para su elaboración están reguladas por el Reglamento sobre el Trabajo de Fin de Título ${ }^{4}$, aprobado en Consejo provisional de Gobierno en su sesión de 23 de septiembre de 2010, y modificado posteriormente en su sesión de 17 de abril de 2013. Este documento tiene como finalidad unificar criterios y procedimientos en torno a la organización y evaluación de estos trabajos con la intención de garantizar la igualdad de derechos y deberes para el conjunto de estudiantes de la Universidad Internacional de Valencia.

\footnotetext{
3 Plan de estudios del Máster Universitario en Formación del Profesorado de la VIU (https://www.universidadviu.es/master-profesorado-secundaria-cap/).

${ }^{4}$ Reglamento sobre el trabajo de fin de título de la VIU (https://www.universidadviu.es/normativa/).
} 


\section{LA DEFENSA PÚBLICA EN UN ENTORNO VIRTUAL, ¿POR QUÉ CAUSA RESPETO A LOS ESTUDIANTES?}

Si las nuevas tecnologías de la información y comunicación forman parte de nuestras vidas, no es de extrañar que su utilización sea preceptiva en una universidad que imparte docencia en modalidad online. Si además, consideramos que la asignatura de TFM debe tener una marcada orientación profesional y aproximarse a la realidad docente actual, tiene sentido que se exija a los estudiantes que realicen la defensa en un entorno virtual, ya que deben familiarizarse con un medio que convive, desde hace años, con la enseñanza presencial.

Si bien muchos de ellos sí lo están, otros carecen de la competencia tecnológica que requiere dominar este entorno, y este hecho les genera cierto respeto ante una prueba que precisa de preparación y práctica. Ahora bien, una vez superada, reconocen que esta materia les aporta no solo los conocimientos y aptitudes necesarias para el ejercicio docente, sino también una habilidad tecnológica avanzada que les diferencia.

En este sentido, entre nuestras responsabilidades como tutores académicos, se encuentra la de formar al estudiantado en el manejo de la plataforma Blackboard Collaborate Ultra, concretamente en el funcionamiento y desarrollo de una defensa pública por videoconferencia. El estudiante debe conocer y dominar esta herramienta hecho que se presupone, ya que previamente ha cursado los módulos teóricos exigidos, así como los formalismos técnicos del acto de presentación (tiempo de antelación con el que deberá conectarse, encendido de la cámara, configuración del sonido, pase de diapositivas, control del tiempo, asignación de permisos de presentador, desplazamiento entre salas virtuales, etc.). Con esta información se sentirá más cómodo y podrá centrarse en la defensa, lo cual redundará en la calidad de su exposición y, por consiguiente, en el resultado final de la prueba.

En cualquier caso, las indicaciones del tutor no deberán limitarse a la parte técnica del proceso. Es importante que el estudiante conozca también los criterios de evaluación (González-Calero \& Turégano, 2012), las rúbricas utilizadas por la comisión evaluadora y el proceso de deliberación y retroalimentación. Cuanta más información disponga sobre el desarrollo de la prueba, mayor seguridad y menor ansiedad le producirá enfrentarse a ella. Corresponde, pues, al tutor preparar a los estudiantes para superar este reto académico y minimizar posibles miedos ante una prueba oral que evidencia el esfuerzo realizado durante duros meses de trabajo (San Mateo-Valdehíta, Escobar-Álvarez \& Chacón-Beltrán, 2018).

\section{DESARROLLO DE LA EXPERIENCIA DE INNOVACIÓN: ESTRATEGIAS Y BUENAS PRÁCTICAS IMPLEMENTADAS EN LAS ÚLTIMAS EDICIONES DEL MÁSTER.}

A lo largo de las últimas ediciones del Máster Universitario de Formación del Profesorado se han ido adoptando una serie de medidas organizativas que, poco a poco, han ido moldeando la asignatura de TFM que se imparte hoy en día en esta universidad. De manera progresiva y atendiendo a la experiencia que cada edición del máster nos aporta, se han ido sistematizando documentos de trabajo (plantillas, informes de autorización, compromisos de aceptación de dirección, etc.), criterios de evaluación, materiales docentes y procesos de tutorización, entre otros muchos aspectos. Se han ido implementando iniciativas que han funcionado positivamente y otras que todavía podrían mejorarse, pero todas ellas han servido para recabar información sobre el proceso de aprendizaje en esta materia y mejorar la calidad de la formación que reciben nuestros estudiantes en el marco de estas enseñanzas.

A continuación, compartiremos algunas prácticas que hemos ido incorporando en las distintas fases de preparación y defensa pública del trabajo de fin de máster; 
cinco fases por las que atraviesa el alumnado desde que empieza a planificar esta prueba hasta que se le comunica su calificación final:

- Planificación y organización de la prueba.

- Preparación del acto de presentación y defensa.

- Acto de presentación y defensa pública.

- Evaluación por parte de la comisión evaluadora.

- Retroalimentación y comunicación de la calificación final.

Si bien estas fases no aparecen detalladas en el programa de la asignatura, se trabajan progresivamente para que el estudiante se sienta acompañado en todo momento. En este sentido, la intervención del tutor no debería limitarse, pues, a la etapa de redacción del TFM, sino que debería incluir el acompañamiento del alumnado hasta el día de su defensa. Las iniciativas que compartiremos en cada una de estas fases servirán de ayuda tanto a los estudiantes que se enfrentan a esta prueba por primera vez como a los profesores nóveles que les dirigen.

\subsection{Planificación y organización de la prueba}

La fase de planificación y organización del acto de presentación y defensa es una de las más importantes del proceso porque garantiza que se cumplan satisfactoriamente el resto de fases. Sin una buena planificación será complicado alcanzar los objetivos exigidos por el programa y, por tanto, obtener un buen resultado final.

Con frecuencia, encontramos estudiantes que presentan excelentes trabajos escritos, bien fundamentados y perfectamente publicables, pero que llegan a la fase de defensa sin haber invertido el tiempo necesario en su preparación. Esto ocurre porque olvidan planificarla con la antelación suficiente, viéndose obligados a centrarse en el documento-producto que utilizarán durante su presentación (el documento en Powerpoint o pdf que mostrarán ante el tribunal). Concentran sus esfuerzos en la parte escrita de la asignatura y se relajan tras el depósito oficial del trabajo, olvidando que el tribunal, si bien toma en consideración la calidad y desarrollo del trabajo presentado, lo que evalúa, en realidad, es su exposición y, por tanto, un porcentaje importante de su calificación final dependerá de ello. El estudiante debería conferir a este acto la importancia que merece si quiere conseguir un buen resultado.

Se recomienda, pues, al estudiantado adelantar la preparación de la defensa siempre y cuando su trabajo escrito haya sido revisado y haya recibido el informe de autorización por parte del tutor-, planificándola en paralelo con la etapa final del trabajo escrito. Dado que el plazo entre el depósito oficial y la defensa es bastante reducido (aproximadamente una semana), el estudiante debería anticipar, en la medida de lo posible, la preparación de dicha prueba, algo que no siempre resulta factible debido a la importante carga de trabajo que implica esta materia. No obstante, aquellos que lo consiguen, se enfrentan a la defensa con mayor determinación y tranquilidad, ya que sienten que han ganado unos días para practicarla, familiarizarse con el entorno digital y corregir, con la ayuda del tutor, posibles errores en la exposición.

Por experiencia, los estudiantes que preparan su defensa tan solo unos días antes corren el riesgo de no disponer del tiempo suficiente para practicarla. Por eso, es importante que el tutor planifique con ellos esta etapa y les oriente sobre cómo afrontarla. Como estrategia, se recomienda establecer un cronograma de trabajo real para que el estudiante sea consciente del tiempo del que dispone y sepa cómo 
optimizarlo; un cronograma que puede presentarse a través de una línea en el tiempo, un calendario de trabajo o una representación gráfica de doble entrada (actividades/periodo de tiempo estimado). Lo importante de este ejercicio es que el estudiante dedique unos minutos a planificar el proceso de preparación de la defensa, marque una serie de hitos a corto plazo y visualice su propio avance. Habitualmente cuando se les propone esta actividad, su aceptación es un tanto relativa (muchos estudiantes consideran que se trata de una tarea adicional poco práctica), pero una vez ven plasmados sus objetivos y, sobre todo, se dan cuenta del resultado, reconocen el valor de esta herramienta. Por eso, alentamos a los directores de TFM a adoptar esta práctica con su alumnado porque, sin duda, les ayudará a planificar y optimizar su tiempo.

Asimismo, es interesante que, en esta primera fase, el tutor proponga una videoconferencia a sus estudiantes; una conexión voluntaria en grupo que quedará grabada en la plataforma para su posterior visualización. Durante esta sesión, el tutor les recomendará bibliografía y material de apoyo sobre la presentación de un trabajo académico ante un tribunal, les indicará algunas técnicas de comunicación efectiva y les informará sobre el funcionamiento de la plataforma o el diseño del documento que utilizarán durante su exposición. También les invitará a consultar los recursos digitales disponibles en la Biblioteca Virtual del Campus y les recordará cómo deben citar correctamente las fuentes consultadas, no solo en sus trabajos escritos, sino también en el documento que utilizarán en su presentación. Los estudiantes, por su parte, compartirán ideas, dudas e inquietudes con el resto de sus compañeros. Con esta iniciativa se pretende que el estudiante tenga pleno conocimiento del acto de defensa antes de iniciar su preparación y cuente con las herramientas necesarias para hacerlo. Es, además, una oportunidad para crear un espacio de comunicación más cercano entre el tutor y sus estudiantes.

En línea con lo anterior, cabe añadir que, como novedad en la última edición del máster (abril 2019), se les propuso realizar una pequeña dinámica de grupo durante esta sesión. Se les pidió que, de los alumnos conectados, la mitad adoptaran el rol de tribunal y la otra mitad, el de estudiantes que iban a defender su TFM. De manera improvisada, debían simular los primeros minutos de una defensa, mientras que el resto evaluaba su intervención y realizaba posteriormente una crítica constructiva. A pesar de ser una dinámica un tanto arriesgada por no haberles solicitado preparación previa, el resultado fue muy satisfactorio para el grupo. Esta actividad les ayudó a conocer los criterios de evaluación que el tribunal tiene en consideración para evaluar la defensa, a ser críticos consigo mismo y a darse cuenta de que la manera en la que nos comunicamos es tan importante como el contenido que transmitimos.

Si bien es una iniciativa que se realiza desde hace poco tiempo, su acogida ha sido muy positiva y se seguirá implementando en futuras ediciones.

\subsection{Preparación del acto de presentación y defensa}

Si durante el proceso de elaboración y redacción del trabajo escrito los estudiantes han realizado una tarea de análisis y desarrollo, en la fase de preparación de la defensa, deberán hacer un esfuerzo inverso (Da Cunha, 2016; García \& Martínez-Clares, 2012), es decir, deberán sintetizar contenidos dado que tendrán una limitación temporal de 15 minutos para su presentación.

Para ello, se les recomienda que identifiquen los aspectos más relevantes y originales de su proyecto, resumiéndolos en aproximadamente 20 páginas 0 diapositivas (entre las que se incluirán la portada, la contraportada, el índice de contenidos y las referencias bibliográficas). Utilizar un número superior, supondría explicar el trabajo con rapidez sin profundizar en los apartados más relevantes; por el contrario, utilizar pocas diapositivas incitaría al estudiante a finalizar su presentación con 
antelación, lo cual, sería penalizado por el tribunal. De ahí la importancia de gestionar bien el tiempo, optimizarlo y concentrarse en los aspectos más destacables del proyecto para transmitirlos sin divagaciones.

Una vez seleccionados los contenidos, el estudiante se centrará en el documento-producto que presentará ante el tribunal, el cual no debe contener, por requisitos técnicos de la plataforma, transiciones, animaciones o un número excesivo de imágenes. Dicho documento debe enviarse a través del Campus Virtual, 48 horas antes de la defensa oficial para que los miembros del tribunal puedan consultarlo.

Como apartados básicos se sugiere que la presentación incluya una introducción y justificación del proyecto, objetivos y contenidos, metodología utilizada, criterios de evaluación, una breve descripción de las unidades didácticas, algunos ejemplos de actividades creadas o adaptadas por el estudiante, las competencias transversales y posibles adaptaciones curriculares (atendiendo a la diversidad en el aula), así como las principales conclusiones y limitaciones del proyecto. Todo ello sin necesidad de incorporar mucho texto en cada diapositiva, sino solo algunas palabras clave que le permitan desarrollar sus ideas y hacer que su discurso progrese con naturalidad. Se trata, por tanto, de evitar una lectura del documento preparado y de explicar con sus propias palabras el trabajo personal realizado.

En cuanto a la exposición, se recomienda que el estudiante la prepare con atención, evitando al máximo la improvisación. Paradójicamente, y aunque esta afirmación resulte evidente, todavía existen estudiantes osados que se enfrentan a la defensa sin preparación alguna. Como directores de TFM deberíamos evitar que se dieran estas circunstancias, ya que ni ayudan al estudiante ni a los miembros del tribunal que evalúan su intervención. Por eso, como estrategia de aprendizaje, es interesante proponerles que se graben a sí mismos a través de aplicaciones como Appear in, Audicity, Bossjock Jr. o Screen-O-Matic, visualicen su actuación e identifiquen posibles aspectos de mejora. Pueden hacerlo de manera individual o en grupo, apoyándose en el tutor o incluso en amigos y familiares con experiencia en situaciones similares. Independientemente del apoyo elegido, lo importante es que reciban retroalimentación de una persona de su confianza y que, juntos, identifiquen las debilidades y fortalezas de su presentación. Esta experiencia, además de aportarles una gran cantidad de información (tanto en relación a la comunicación verbal como no verbal), les aporta el refuerzo positivo y la seguridad que necesitan para afrontar con éxito esta prueba.

En coherencia con lo anterior, cabe indicar que el rol e implicación del tutor en esta fase es fundamental no solo desde el punto de vista académico, sino también emocional. El estudiante necesita sentir y estar convencido de que, gracias a su ayuda, ha creado un buen proyecto, un trabajo original y aplicable al ámbito profesional, y que sus ideas merecen ser transmitidas. Por ello, cuanto mayor sea el apoyo recibido por el tutor -no solo en esta etapa, sino a lo largo del proceso de tutorización-, mayor seguridad mostrará el estudiante el día de su defensa ante la comisión evaluadora.

\subsection{Acto de presentación y defensa pública}

La presentación y defensa pública de un TFM es un acto que si se prepara previamente, debería llegar a disfrutarse e incluso a recordarse durante mucho tiempo. A priori, el hecho de que se realice en un entorno virtual con un tribunal en la distancia puede generar cierta intranquilidad, pero cuando el estudiante comienza a hablar sobre aquello que conoce y ha preparado, el miedo a lo desconocido se disipa rápidamente. Si el 
acceso a la sesión se realiza con normalidad y el estudiante se conecta con antelación para comprobar que tanto la cámara web como el audio funcionan correctamente, no debería producirse incidencia alguna.

Tras la defensa, se abre un turno de intervenciones para los miembros del tribunal, momento en el que se plantearán al estudiante algunas preguntas sobre su trabajo, a las que podrá contestar en el tiempo que estime necesario. Al finalizar, el tribunal se desplazará a una sala virtual distinta para consensuar el feedback y la nota final, mientras que el estudiante esperará en la sala principal. Una vez acordado, se le comunicará su valoración y la calificación global de la asignatura.

Dado que la defensa es un acto público, cualquier director de TFM o estudiante del máster (de la edición en curso) puede asistir. Es más, es recomendable invitar a nuestros tutorandos a presenciar la defensa de alguno de sus compañeros (ya sea síncronamente o asíncronamente) antes de realizar la suya propia, ya que esta experiencia les proporciona una visión más completa del acto académico. Por su parte, el tutor también puede asistir a las defensas de sus estudiantes (haya sido convocado o no como miembro del tribunal), pero si actúa como miembro no podrá emitir un juicio valorativo u otorgar una calificación sobre su intervención.

En realidad, la defensa de un TFM es un acto sencillo en el que el estudiante expone el trabajo que, junto a su tutor, ha realizado durante los últimos meses. Afortunadamente el estudiantado se interesa cada vez más y con mayor antelación por este acto, lo cual es muy positivo. Piensan en esta prueba durante la etapa de redacción del TFM porque visualizar ese momento les ayuda a seleccionar mejor los contenidos que luego explicarán ante el tribunal. Solicitan al tutor información sobre cómo prepararla, sobre su desarrollo y sobre los criterios de evaluación. Son, pues, más conscientes de que, si bien esta asignatura se compone de un módulo escrito y otro práctico (el trabajo escrito y la defensa), ambas actividades están interconectadas y no pueden trabajarse de manera separada. Por eso, sería interesante que, al igual que el estudiante tiene acceso a algunos modelos de TFM que le sirven de guía para preparar su proyecto escrito en la plataforma, también pudiera facilitársele algún ejemplo de defensa real que le sirva, no tanto como modelo a seguir (ya que cada presentación es distinta), sino como punto de partida para realizar la suya propia. Sería una iniciativa que beneficiaría a muchos estudiantes del máster e incluso a muchos tutores que participan por primera vez como miembros de un tribunal académico y desconocen los formalismos de este acto.

\subsection{Evaluación por parte de la comisión evaluadora}

La evaluación del acto de presentación y defensa se realiza principalmente en base a dos fuentes de información: el informe de evaluación del tutor y el propio acto de defensa.

En el informe de evaluación, el tutor indica el nombre completo del alumno, su propio nombre y apellidos, el itinerario (en nuestro caso, lengua extranjera), el título del TFM, la convocatoria, la calificación propuesta y su justificación, el porcentaje de plagio resultante del análisis del trabajo a través de las herramientas antiplagio, las fortalezas y debilidades del proyecto y otros comentarios pertinentes que considere puedan ayudar al tribunal a tener más información sobre el proceso de tutorización del estudiante (número de envíos y revisiones, interés y compromiso, aceptación de modificaciones, etc.). Dicho informe no exime a los miembros del tribunal de la lectura del trabajo escrito, sino que es una herramienta más de información en la evaluación final.

Por lo que respecta a la defensa, tras la exposición del estudiante, los miembros del tribunal comparten opiniones y recomendaciones de mejora, acuerdan la retroalimentación que comunicarán al estudiante y deciden la calificación final, una 
decisión que no siempre resulta fácil. De ahí la importancia de utilizar una rúbrica de evaluación detallada que permita resolver posibles disparidades entre los miembros de la comisión evaluadora; una información que no suele compartirse con el alumnado, siendo un error que debería subsanarse para que todos los agentes implicados (estudiantes, evaluadores y centro) dispusieran de las mismas «reglas del juego».

No tiene sentido que el estudiante que va a realizar una prueba con la que concluirá sus estudios de postgrado, no tenga conocimiento de los criterios sobre los que será evaluado. Por eso, sería interesante empezar a compartirla e, incluso, a consensuarla. Autores como Pinilla (2012, citado en Onieva, 2016, p. 190) consideran que las rúbricas:

«no [son] herramienta[s] democrática[s] ni participativa[s], porque a pesar de su importancia, no ha[n] sido consensuada[s] con los estudiantes para ayudarles en su aprendizaje y evaluación. De esta manera los alumnos no logran entender ni comprender exactamente cómo han de incorporar a sus exposiciones los criterios de evaluación que se les exige».

Si estas herramientas no se consensuan ni se comparten con el estudiante, su utilidad en la evaluación se reduce considerablemente, convirtiéndose así en un instrumento poco efectivo y de uso unidireccional. El potencial de las rúbricas se encuentra precisamente en dicha explicitación de criterios, hecho que contribuye a la mejora del aprendizaje y al rendimiento de los estudiantes (Estapé-Dubreuil et al., 2012).

Esta reflexión ha sido también abordada en estudios recientes como el de San Mateo-Valdehíta et al. (2018), quienes analizan las necesidades reales de los estudiantes que cursan estas asignaturas y las dificultades a las que se enfrentan. $Y$ entre las señaladas, se indica la relativa a la evaluación, una laguna académica que las universidades todavía deben explorar en profundidad. En su estudio, el alumnado encuestado $(n=434)$ indica que «no se detallan suficientemente los criterios de evaluación del trabajo escrito y de la defensa» (p. 313) y, por tanto, reclaman información. El desconocimiento sobre los aspectos evaluables les genera dudas, preocupación e incertidumbre, una situación que podría resolverse si se les facilitara la rúbrica de evaluación que tanto el tutor (en el trabajo escrito) como los miembros del tribunal (en el acto de la defensa) utilizan para otorgar la calificación.

Por eso, como tutores de esta materia, apostamos porque dichos criterios y su correspondiente peso dentro de la evaluación se pongan a disposición del estudiantado a través de un documento consensuado al que tengan acceso en el Campus Virtual o como anexo a la guía docente de la asignatura. Esta iniciativa garantizaría no solo la equidad en las valoraciones de los tribunales, sino también la homogeneización de los criterios mediante los que se les evalúa.

Para ello, se hace necesario seguir impulsando un cambio en los procedimientos de evaluación de la defensa que permitan unificar criterios y minimizar las discrepancias subjetivas que pudieran darse entre los distintos tribunales evaluadores (Sanz Miguel, 2015), pero también que nos permitan consensuar democráticamente con los protagonistas de este proceso de aprendizaje los criterios más justos por los que deberían ser evaluados. Mejorar la rúbrica utilizada por la comisión evaluadora y compartirla con los estudiantes en las próximas ediciones es un reto en el que estamos ya trabajando. 


\subsection{Retroalimentación y comunicación de la calificación final}

La última fase de la defensa es quizás la más interesante para el estudiante, ya que descubrirá la valoración de su trabajo de acuerdo al criterio de tres profesores especialistas en la materia. Será el presidente del tribunal quien inicie la comunicación del feedback acordado resaltando, por una parte, los aspectos positivos de su proyecto $y$, por otra, aquellos en los que debería prestar más atención en futuros trabajos académicos. A continuación, el resto de miembros del tribunal tomarán la palabra para complementar la información trasladada.

Seguidamente se da paso al estudiante para que comente, matice o amplíe aquello que considere necesario. Tras su intervención, el presidente le comunicará su calificación final que será la combinación de la nota obtenida en el trabajo escrito (otorgada por su tutor) y en la defensa (otorgada por los miembros de la comisión evaluadora). El feedback se comunicará al estudiante siempre desde una perspectiva constructiva y nunca fiscalizadora. El estudiante podrá entonces realizar las observaciones que considere pertinentes sobre la retroalimentación recibida e incluso solicitar por escrito dicha información al Presidente del tribunal.

Finalmente cabe señalar que, en esta fase del proceso, se debería incitar a aquellos estudiantes que han presentado trabajos académicamente destacables a difundir sus resultados y reflexiones, y a publicar sus creaciones. Si bien se trata de un trabajo elaborado para cumplir las exigencias de un programa de máster, es interesante que se motive a los futuros docentes a compartir sus conocimientos y primeras experiencias en el campo de la investigación. Hay estudiantes con ideas brillantes cuyos trabajos deberían ver la luz y no quedarse simplemente almacenados en repositorios universitarios.

\section{RESULTADOS}

Incorporar y sistematizar las propuestas presentadas anteriormente nos ha permitido mejorar el funcionamiento de la asignatura, aumentar la coordinación entre el profesorado y diseñar un proceso de dirección y tutorización de trabajos de fin de máster satisfactorio para el estudiantado. Poner por escrito todas las acciones que se han ido implementando a lo largo de las últimas ediciones nos ha ayudado a reflexionar sobre este proceso y sobre la importancia que tienen estas asignaturas en la formación y desarrollo del futuro docente, así como a identificar nuevas necesidades para conseguir que los estudiantes que cursen estas materias lo hagan con la tranquilidad con la que cursan el resto de asignaturas del programa.

El resultado de la experiencia es, por tanto, positivo principalmente porque:

- Permite al estudiante estar informado sobre el desarrollo de la materia, sobre las fases del proceso de tutorización y defensa, el funcionamiento de la plataforma en el marco de una defensa pública por videoconferencia y los criterios de evaluación del tribunal.

- Permite a los directores nóveles conocer algunas de las prácticas docentes que se llevan a cabo en el máster y cuya aplicación ha sido acertada.

- Permite a los directores con mayor experiencia seguir investigando sobre las mejoras que todavía pueden implementarse para que el proceso de enseñanza-aprendizaje de nuestro alumnado sea lo más cercano posible a la realidad profesional a la que en breve se enfrentarán.

Si queremos formar a estudiantes que van a dedicarse a la docencia, deberemos implicarnos al máximo en el proceso de acompañamiento porque solo así encontrarán sentido a lo que hacen y aportarán valor a esta profesión. 


\section{CONCLUSIONES Y RETOS FUTUROS}

Ciertamente la asignatura de Trabajo de Fin de Máster (TFM) es una materia distinta al resto de asignaturas que cursa el estudiante a lo largo del Máster Universitario de Formación del Profesorado. Si bien implica un reto para el alumnado que debe enfrentarse a un trabajo de iniciación a la investigación aplicado a la realidad profesional y defenderlo ante tres profesores de la especialidad, también lo es para los docentes que deben tener las competencias necesarias para dirigir, tutorizar y evaluar un trabajo de esta envergadura.

A ello se añade una circunstancia que a muchos genera cierta intranquilidad: defender públicamente su proyecto a través de videoconferencia. Aunque, en realidad, el acto de presentación y defensa en un entorno virtual sea, en esencia, el mismo que un acto presencial, es importante que, tanto nuestros estudiantes como el profesorado novel que se inicia en la tutorización de estos trabajos, tengan en cuenta algunos aspectos clave para preparar con éxito dicha prueba (Flores et al., 2013). De ahí la necesidad de compartir experiencias que, siendo positivas en estos estudios, puedan extrapolarse a otras titulaciones e, incluso, servir de referencia a otras universidades.

Partiendo del objetivo inicial de este artículo, el de compartir estrategias y buenas prácticas adoptadas en las últimas ediciones del máster para la preparación de la defensa de TFM a través de videoconferencia, se pretende analizar el desarrollo de estas pruebas en el marco de la enseñanza virtual. Existen numerosos estudios que se centran en la enseñanza presencial (Cabrera et al., 2013), pero se hace necesario también investigar qué ocurre en un contexto virtual, ya que ambas modalidades educativas conviven hoy en día. $Y$ de entre todas las iniciativas compartidas, nos gustaría resaltar una que consideramos deontológicamente esencial: la de informar a nuestros estudiantes sobre lo que se espera de ellos en este acto académico. No podemos ocultarles los criterios de evaluación, las rúbricas, las rejillas o las listas de cotejo (Onieva, 2016) que utilizamos como evaluadores porque estaremos infundiéndoles inseguridad. Los estudiantes son los protagonistas de este proceso y deben conocer todas las reglas del juego para actuar en consonancia; se les debe, pues, invitar a que asistan a las defensas de otros compañeros; apoyarles no solo en la etapa de redacción del trabajo escrito, sino también en la preparación de su presentación; facilitarles un modelo de defensa y proponerles estrategias de retroalimentación y autoevaluación que dinamicen y mejoren este proceso.

En cuanto a los retos de futuro, nos enfrentamos a la mejora de las herramientas de evaluación, a la posibilidad de iniciar procesos de co-tutorización, a la creación de grupos de discusión entre el profesorado y el alumnado que nos permita identificar posibles disfunciones en el desarrollo de estas materias, al establecimiento de procesos de mentorización para el profesorado novel, a la creación de protocolos académicos ante posibles casos de ciberplagio (Gallent, 2019) y a la mejora de un trabajo colaborativo entre los distintos agentes implicados en esta materia (profesorado, estudiantes y centro), entre otras muchas iniciativas.

En conclusión, a través de este artículo hemos querido reflexionar y generar interés sobre el desarrollo de estas asignaturas en un entorno virtual, sobre la importancia de compartir experiencias docentes que redunden en la mejora de estas materias y sobre la necesidad de seguir investigando en esta línea para que la defensa de un trabajo de fin de máster no suponga un proyecto inalcanzable o desalentador para nuestros estudiantes. 


\section{REFERENCIAS}

Cabrera, N., Pérez, A., Guitert, M., Sangrà, A., Conesa, J., Guàrdia, L., Burguet, M., \& Rodera, A. (2013). Guía para diseñar un TFM. Barcelona: Agència de Gestió d'Ajuts Universitaris i de Recerca, Agaur y UOC.

Cabrera, N., Sangrà, A. \& Rodera, A. (2012). Desarrollar y evaluar competencias a través del TFM. Marco conceptual y guías didácticas. Revista del Congrès Internacional de Docència Universitària i Innovació (CIDUI), 1 (pp. 1-23). Recuperado de: https://bit.ly/2G4grqb

Da Cunha, I. (2016). El trabajo de fin de grado y de máster. Redacción, defensa y publicación. Barcelona: Editorial UOC.

De Pro Bueno, A. J., Sánchez Blanco, G., \& Valcárcel Pérez, M.V. (2013). ¿En qué medida están contribuyendo los TFM a los resultados de aprendizaje planificados?. Revista Eureka sobre Enseñanza y Divulgación de las Ciencias, 10 (núm. extra), 728-748. Recuperado de: https://bit.ly/2Hv8gEa

Estapé-Dubreuil, G., Rullan, M., López, C., Pons i, J. \& Tena, D. (2012). Rúbricas y evaluación de competencias en los TFG. Un paso atrás para saltar. VII Congreso Internacional de Docencia Universitaria e Innovación. La Universidad: una institución de la sociedad. Barcelona. Recuperado de: https://bit.ly/2WQ0V7Y

Flores, C., Martínez, J.A., Martínez M.I., Pascual, N. E., \& Sanz, E. (2013). Manual de orientación para la tutorización y evaluación del trabajo de fin de grado: Facultad de Letras y de la Educación. Logroño: Universidad de La Rioja.

Gallent, C. (2019). El ciberplagio, una asignatura pendiente en la formación del profesorado universitario. Levante-EMV, 10 de mayo de 2019.

García, M. P. \& Martínez-Clares, P. (coords.) (2012). Guía práctica para la realización de Trabajos de Fin de Grado y Trabajos de Fin de Máster. Murcia: Edit.um (Ediciones de la Universidad de Murcia).

González-Calero, J. A. \& Turégano, P. (2011). Reflexiones sobre el desarrollo y la evaluación de Trabajos Fin de Máster en el Máster Universitario en Profesor de Educación Secundaria Obligatoria y Bachillerato en la especialidad de matemáticas por la UCLM. Libro de actas, VII Intercampus 2011. Recuperado de: https://bit.ly/2UPbXgF

Hernández-Leo, D., Moreno, V., Camps, I., Clarisó, R., Martínez-Monés, A., MarcoGalindo, M.J., \& Melero, J. (2013). Implementación de buenas prácticas en los trabajos de fin de grado. Revista de Docencia Universitaria (REDU), 11 (núm. especial), 269-278. DOI: https://doi.org/10.4995/redu.2013.5556

Onieva López, J. L. (2016). Estrategias didácticas y recomendaciones para la defensa oral de los Trabajos de Fin de Grado y Fin de Máster. Revista Electrónica Interuniversitaria de Formación del Profesorado, 19 (3), 185-198. DOI: http://dx.doi.org/10.6018/reifop.19.3.206331

San Mateo-Valdehíta, A., Escobar-Álvarez, M.A., \& Chacón-Beltrán, R. (2018). El trabajo de Fin de Máster (TFM) de Humanidades en el EESS: Análisis de las necesidades de los estudiantes. Revista de docencia universitaria (REDU), 16 (1), 303-319. DOI: https://doi.org/10.4995/redu.2018.9873

Sanz Miguel, M. C. (2015). Desarrollo e implementación de una herramienta de evaluación en los tribunales de los trabajos de fin de grado y fin de máster en las ciencias de la salud. Proyecto de Innovación y Mejora de la Calidad docente, $\mathrm{n}^{\circ}$ PIMCD207, Universidad Complutense de Madrid. Recuperado de: https://eprints.ucm.es/34951/

Sayos, R., Amador, J.A. \& Pagés, T. (2016). Los trabajos de fin de grado en la Universidad de Barcelona. Barcelona: Ediciones Octaedro y ICE.

Serrano, R. \& Pontes, A. (2015). Nivel de desarrollo de las competencias y objetivos generales del Máster Formación del Profesorado de Enseñanza Secundaria. Perfiles educativos, 150 (37), 39-55. Recuperado de: https://bit.ly/2lhsgeX 
Universidad Internacional de Valencia (VIU) (2019). Plan de estudios del Máster Universitario en Formación del Profesorado. Recuperado de: https://www.universidadviu.es/master-profesorado-secundaria-cap/

Universidad Internacional de Valencia (VIU) (2019). Reglamento sobre el Trabajo de Fin de Título. Recuperado de: https://www.universidadviu.es/normatival

Viejo, C. \& Ortega, R. (2018). Competence for research: the master's thesis and its scientific training potentiality. Revista de Innovación y buenas prácticas docentes, 5, 46-56. DOI: https://doi.org/10.21071/ripadoc.v5i.10970 